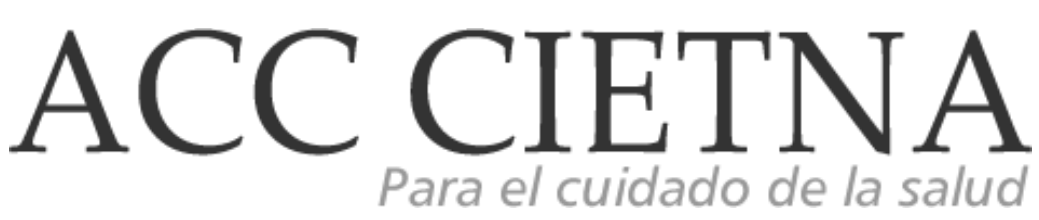

\title{
Contribuciones significativas de la fenomenología de Edmund Husserl, Martin Heidegger y Hans- Georg Gadamer a la investigación en enfermería
}

\section{Significant contributions of Edmund Husserl, Martin Heidegger, and Hans- Georg Gadamer's phenomenology to nursing research}

\author{
Lucila Acevedo-Figueroa 2, a, b \\ I.acevedofigueroa@ugto.mx \\ https://orcid.org/0000-0001-6471-684X
}

José Alberto Agüero-Grande 2, a jaguero@uabc.edu.mx https://orcid.org/0000-0002-2006-3805

Andrea Ximena Oyola-Lamprea *, 1, a

oyola.a@javeriana.edu.co

https://orcid.org/0000-0003-0095-8086

Yolanda Ivonne Trujillo-León ${ }^{2, ~ a ~}$

yi.trujilloleon@ugto.mx

https://orcid.org/0000-0001-9569-3345

\author{
* Autor corresponsal \\ ${ }^{1}$ Pontificia Universidad Javeriana, Bogotá, Colombia \\ 2 Universidad de Guanajuato, México \\ a Licenciado en enfermería \\ b Magister en Ciencias de Enfermería
}

Fechas importantes

Recibido: 2021-06-29

Aceptado: 2021-11-15

Publicado online: 2021-12-17

\section{Resumen}

La disciplina del cuidado es la expresión del trabajo afectivo, acreditado y competente, que el profesional de enfermería efectúa en la interacción con la persona, de acuerdo a su temporalidad y en diferentes contextos en donde se desarrolla o en donde procura su salud; al proporcionar cuidado se concibe a la persona como la unidad esencial del cuerpo que le permite mediar entre la naturaleza, la cultura y el espíritu, como ser único inseparable y particular; consecuentemente se alude que la tarea del cuidar está infundida por la filosofía, porque implica el análisis de los propósito de la vida humana, de la naturaleza del ser y de la realidad, de los valores presentes en el cuidado y el discernimiento intelectual para el bienestar de la persona. Para propugnar esta idea, se hace el acercamiento a algunos filósofos cuyo referente han discurrido sobre la investigación en la disciplina del cuidado, tales como Edmund Husserl, Martin Heidegger y Hans-Georg Gadamer enfocados a develar el real significado de la experiencia vivida con un interés genuino y respetuoso por su existencia y fragilidad.

Palabras clave: Fenomenología, Edmund Husserl, Martin Heidegger, Hans-Georg Gadamer, Investigación en enfermería 


\begin{abstract}
The discipline of care is the expression of the affective, accredited and competent work that the nursing professional carries out in the interaction with the person, according to their temporality and in different contexts where they develop or where they seek their health; When providing care, the person is conceived as the essential unit of the body that allows it to mediate between nature, culture and spirit, as a unique, inseparable and particular being; Consequently, it is alluded that the task of caring is infused by philosophy, because it involves the analysis of the purpose of human life, the nature of being and reality, the values present in care and intellectual discernment for well-being of the person. To advocate this idea, the approach is made to some philosophers whose referents have discussed research in the discipline of care, such as Edmund Husserl, Martin Heidegger and Hans-Georg Gadamer focused on revealing the real meaning of the experience lived with an interest genuine and respectful for its existence and fragility.
\end{abstract}

Keywords: Phenomenology, Edmund Husserl, Martin Heidegger, Hans-Georg Gadamer, Nursing Research.

\title{
Introducción
}

Es imperioso mencionar los primeros aportes a la investigación en enfermería fueron realizados por Florence Nightingale en el siglo XIX en el año 1859 cuando gracias a sus conocimientos sobre matemáticas y estadística pudieron ser aplicados a la disciplina de enfermería; gracias a esta contribución, en décadas posteriores se han podido evidenciar múltiples investigaciones de enfermeras quienes motivadas por el deseo de mejorar sus cuidados a los pacientes mediante la evidencia científica, han desarrollado diversidad de modelos y teorías basadas en la investigación, la cual en principio fue orientada exclusivamente por la corriente positivista. Este enfoque al empirismo en principio contribuyó enormemente al fortalecimiento del cuerpo de conocimientos de enfermería y a su desarrollo como disciplina científica ${ }^{1}$.

Adicionalmente, con el surgimiento de múltiples enfermeras investigadoras y el deseo de brindar cuidados basados en la evidencia científica, motivó a que la investigación tomará un rumbo distinto a lo netamente tangible con el fin de poder dar explicación a los fenómenos observables y poder así dar explicaciones y comprender mejor a los individuos o al colectivo, en diferentes contextos generando un acercamiento y un cuidado más humanizado en el proceso de la atención de enfermería.

De acuerdo a esto, se encontró en la fenomenología y en la hermenéutica una oportunidad para poder orientar las investigaciones posteriores. Dentro de esta metodología se han tenido en cuenta diversos exponentes quienes fueron precursores de lo que se conoce hoy como la fenomenología como Edmund Husserl y Martin Heidegger y entre otros pensadores como Hans-Georg Gadamer, quienes llevaron a cabo diversos aportes los cuales fortalecieron esta tendencia logrando convertirse así en importantes referentes filosóficos. Posteriormente, alrededor de los años 70 hasta la época, hubo un importante aumento de múltiples investigaciones cualitativas en enfermería y en el desarrollo de estudios con enfoques 0 diseños cualitativos orientados a la fenomenología y hermenéutica ${ }^{2}$.

La enfermería es una disciplina humanística caracterizada por el cuidado del individuo durante su proceso salud-enfermedad, que requiere de metodologías de investigación que sean capaces de acercarla a los fenómenos que acontecen en su práctica tal y como se presentan y la manera en cómo se experimentan por la misma persona. Razón por la cual esta disciplina se apoya en la fenomenología y hermenéutica para lograr un acercamiento a las experiencias y sus significados y cómo llegan a ser interpretados; permitiendo un análisis crítico y reflexivo sobre la importancia e impacto de la enfermería cuando ejerce el cuidado ${ }^{1,3}$. 
Por ello, resulta conveniente llevar a cabo un ensayo que permite reflexionar sobre las contribuciones y aportes que ha tenido la fenomenología y hermenéutica en el desarrollo y fortalecimiento del campo de conocimientos y evidencias científicas de la disciplina de enfermería y como le ha permitido conocer y comprender los fenómenos que surgen en el desarrollo de su ejercicio asistencial y de cuidado.

\section{Desarrollo}

El referencial fenomenológico va al encuentro del significado de la experiencia vivida, asiste a la búsqueda de la comprensión del hombre, sujeto del cuidado y viene, por tanto, al encuentro de la finalidad de la disciplina de enfermería.

Los abordajes fenomenológicos tradicionales son: Fenomenología trascendental o descriptiva, que estudia la estructura esencial del fenómeno (Husserl, Eugen Fink, Van Breda); Fenomenología existencial (Martin Heidegger, Sartre, Merleau-Ponty, Gabriel Marcel), Fenomenología hermenéutica (Martin Heidegger, Gadamer, Ricoeur), mismos enfoques que pueden ser utilizados como referente filosófico y como enfoques de análisis fenomenológico 4,5 .

\section{Pensamiento de Edmund Husserl}

Cuando observamos el nombre de Edmund Husserl en el contexto de la disciplina de enfermería, recordamos el enfoque fenomenológico y la metodología cualitativa. Husserl no sólo es considerado como el fundador de la fenomenología, su amplio desarrollo conceptual contribuye a la desaparición del positivismo el cual inspiró enfoques fructíferos a las ciencias sociales.

En este bagaje de inspiración, para Husserl en 1965, los objetos se convierten en fenómenos a medida que son percibidos, recordados, representando pictóricamente, imaginado, identificado, distinguido, recibido, opinado, evaluado, etc. ${ }^{6}$.

El "Conocimiento" para Husserl es obtenido al aprehenderlas esencias de la experiencia consciente la presencia de la persona que experimenta los actos conscientes. El soporte implica suspender el juicio sobre el mundo natural y su existencia. Husserl cree en el análisis de la experiencia consciente. La experiencia debe realizarse desde un punto de vista en primera persona. De la misma forma una perspectiva en primera persona, uno no puede estar seguro de que el objeto percibe experiencias (por ejemplo, una mesa) aparte de la experiencia en sí mismo. Por esta razón, Husserl afirma que los supuestos sobre la existencia de objetos de experiencia fuera de la experiencia (p. ej., objetos físicos) deben estar suspendidos.

La reducción eidética en esta primera forma de reducción se trata de la diferencia de actitud de la experiencia y sobre lo general en la experiencia, implica la identificación y eliminación de cualquier característica de nuestras experiencias para intuir las características invariantes y necesarias de la experiencia 6,7 .

Intuir las características o esencias básicas de nuestra experiencia como personas llámese enfermera o persona que recibe el cuidado procede a través de lo que Husserl en (1997) llama a la variación libre en imaginación. Las características esenciales en un objeto en la experiencia consciente son esencias que no se pueden cambiar y sin el cual la experiencia no sea la experiencia que es. En la fenomenología husserliana, la esencia del significado real del fenómeno se logra a través de "la primera persona que experimenta el fenómeno"8. 
En este sentido el descubrir o redescubrir el sentido existencialmente en aquello que es considerado como teoría, concepto, definiciones, procedimientos preestablecidos, relaciones de causa y efecto, hipótesis, conjeturas, entre otros. Reflexionar en el experienciar es fundar lo que se manifiesta fenomenológicamente buscando el sentido y esencia de la forma como se muestran en cualquier contexto de salud, captar la experiencia vivida, nutriendo el conocimiento, con el propósito de mejorar el cuidado que se les entrega a las personas en cualquier contexto, pudiendo ser este cada vez más integral y humanista, mejorando el autoconocimiento en la realización del mismo ${ }^{9}$.

Enfermería busca dentro del fenómeno del cuidado quizá sin saberlo una actitud fenomenológica que invite a dejar que las cosas aparezcan con sus características propias, como ellas son, dejando que la esencia se manifieste en la conciencia del sujeto, con el objetivo de no transformar, no alterar la originalidad de los fenómenos y poder entonces establecer estrategias sobre el cuidado realizado en la interacción de dos seres al final de cuenta en un mismo escenario y temporalidad ${ }^{10}$.

Es así como la fenomenología trascendental o descriptiva considera una tarea de clarificación para poder llegar a las cosas mismas partiendo de la propia subjetividad, en cuanto las cosas se experimentan primariamente como hechos de conciencia, cuya característica fundamental es la intencionalidad ${ }^{10,11}$.

Soto Núñez y Vargas Celis afirman que la fenomenología de Husserl se aproxima a lo propiamente humano, y la disciplina de enfermería es humana; ${ }^{12}$ además De la Cuesta manifiesta que este tipo de aproximación produce conocimiento científico que fortalece la práctica de enfermería, y, por tanto, contribuye al desarrollo disciplinar desde el campo de la práctica y de la investigación ${ }^{13}$.

Para conocer la realidad de las cosas Husserl impacta con un método diferente, que utiliza el análisis reflexivo y que implica una responsabilidad social, a través del estudio de los fenómenos en forma pura que anhela y resulta necesario volver ("a las cosas mismas"), no parcializando, ni manipulando al objeto de estudio como lo hacían las ciencias de la época así lo refiere Thurnher ${ }^{14}$.

Con estas contribuciones se muestra que no se trata de una descripción empírica o meramente psicológica, sino trascendental, esto es, constitutiva del conocimiento de lo experimentado, porque se funda en los rasgos esenciales de lo que aparece a la conciencia. La fenomenología requiere rigurosidad como método de investigación, es un método, que le entrega a enfermería la oportunidad de desarrollar la disciplina, aproximándose en profundidad al fenómeno de interés: el cuidado del ser humano ${ }^{15}$.

\section{Pensamiento de Martin Heidegger}

Martin Heidegger fue uno de los principales discípulos de Husserl, que en 1927 publica su obra el Ser y el Tiempo, en que propone y establece a la fenomenología en una analítica existencialista, lo que permite ver el ser de los entes, poniendo en marcha así una postura ontológica. Heidegger sustituye "conciencia trascendental" por "vida en su factibilidad", lo que se quiere decir a la construcción del camino hacia el mundo de la existencia ${ }^{16}$.

Abonado de esta manera el concepto brinda significado al fenómeno; mostrando así la relación alineada al mundo en su propia comprensión, componiéndose así al mundo de las cosas u 
objetos útiles a la propia existencia; por lo tanto, a diferencia de Husserl en su lenguaje la fenomenología es eidética, para Heidegger es interpretativa ${ }^{13}$.

Para llegar a ello, Heidegger propone la expresión de Dasein que, dentro de su construcción morfológica o etimológica, "Da" es aquí o allí y "Sein" es ser. Transformándose en una reflexión de la existencia en su propia historicidad vinculada a su realidad, de esta manera dando mayor peso a la dimensión ontológica poniéndose de lado la epistemológica, aspecto que mayormente se acentúa en esta fenomenología ${ }^{16}$.

El Dasein está situado en un modo dinámico para lograr ser, no solo es esencia sino existencia, teniendo posibilidades para el ser-ahí, donde el ser es la existencia humana y el ahí es el mundo cual habita; lo que se traduce que existir es estar en el mundo. ${ }^{13}$ Desde esta concepción la existencia humana se abre en su propio mundo, tal como es experimentado, construyendo los significados a las vivencias o modos de vivir y establecer relaciones que va necesitando para continuar siendo. Como lo menciona Heidegger la fenomenología es sobre aprender al ser de los entes donde ese ser es perceptible como el fenómeno, es decir mostrarse en su cómo. En sus palabras, "ver lo que se muestra tal como se muestra por sí mismos" en su sentido ontológico "a las cosas mismas". En este punto es cuando se observa como la fenomenología ve la necesidad de empezar a vincularse con la hermenéutica ${ }^{17}$.

Considerando el objetivo de Heidegger en el que busca entender el significado del ser de los entes y como el mundo es inteligible para ellos; es cuando se comprende el porqué de la propuesta de una fenomenología interpretativa o hermenéutica, convirtiéndose así en un método más allá que un referente filosófico; para lograr su descubrimiento de una manera totalmente distinta a la tradición positivista ${ }^{16,19}$.

En este punto la aplicación de la hermenéutica radica en ser uno mismo en sí mismo, a lo que se quiere decir que cada Dasein logre ser propiamente sí mismo. Encontrándose al Dasein hermenéutico el cual es el que transita en el ser-en-el-mundo, en su entendimiento existencial, una base para una interpretación del ser en general, bajo su estructura originaria y siempre total ${ }^{17}$.

La fenomenología hermenéutica es propuesta por Heidegger para descubrir y develar el significado del sentido del ser. La interpretación no se basa solamente en adquirir un conocimiento sobre algo comprendido, sino al acercamiento a las diferentes líneas generadas en el comprender.

A partir de este punto se puede empezar a revisar como esta perspectiva puede llegar a tener implicaciones en la enfermería. El cuidado es uno de los principales elementos que construyen esta disciplina. Diversos autores concuerdan que su abordaje conceptual es altamente complejo derivado a cuestiones en su fundamentación teórica-epistemológica como en la práctica.

En una revisión realizada por Ramírez, Cárdenas y Rodríguez en donde reflexionan sobre el cuidado de enfermería desde la hermenéutica de Heidegger plantean que "El cuidado en su abordaje más amplio consiste en una forma de ser y estar con otros, posee características existenciales y contextuales (modo de ser) y (estar con), de tal forma que el cuidado se visualiza en dos dimensiones: fenomenológica y holística, es decir, entender los significados que la persona le confiere a sus experiencias en interacción con la enfermera(0)"17. 
Por otro lado, Soledad y Herrera, en su artículo Fundamentos fenomenológicos para un cuidado comprensivo, se propone como desde la ontología de Heidegger, los seres humanos están comprometidos con las cosas que tienen significado y las valora porque reconoce potencialidades para ellos dentro de su mundo ${ }^{18,19}$.

Por lo tanto, el cuidar de manera comprensiva es percibir intencionadamente ese mundo construido por las redes de significados socioculturales atribuidos a la situación de los procesos salud-enfermedad que determinará el cuidado.

Desde esos planteamientos, a través de la aplicación de la fenomenología interpretativa de Heidegger, se logra visualizar la experiencia humana y la construcción de los significados intersubjetivos que se construyen alrededor del cuidado desde su propia realidad. Representa una oportunidad sumamente importante para comprender el ejercicio de la enfermería y transformarse para lograr procesos de cuidados más eficaces y mejorar la relación enfermera(o)-paciente.

\section{La Hermenéutica Gadameriana}

La hermenéutica parte del griego hermenéuticos, que traduce "interpretación" y este término se asocia a su vez con la interpretación o comprensión, de textos escritos o realidades y se encuentra desligada de la fenomenología. En principio, este se encontraba estrechamente relacionado con la comprensión de textos sagrados, pero a través del tiempo fue acuñado también a la filosofía y a las ciencias humanas y sociales ${ }^{20}$.

Adicionalmente Pérez Vargas, et. al. en su análisis, define los conceptos de hermenéutica como el método que busca una interpretación coherente de un todo donde el investigador se introduce en el contenido y la dinámica de la persona estudiada; mientras que la fenomenología "se centra en el estudio de las realidades vividas" respetando por completo el relato que se hace la propia persona de sus vivencias.

Para Hans-Georg Gadamer, quien fuera discípulo de Heidegger y posteriormente continuará con la hermenéutica fenomenológica, el ser humano es un ser histórico, por lo cual es necesario tener una conciencia histórica en el acto de investigar pues esta es una parte esencial de nuestro existir ${ }^{21}$.

De este modo, Gadamer afirma que nuestra propia historicidad influye en el acto de investigar, y la propia investigación misma es vista según él como un juego en el cual se debe contar con reglas que guían el flujo de nuestra investigación. Así pues, afirma Gadamer que la hermenéutica no puede ser concebida como una técnica sino como una conciencia de la propia historicidad.'

Por otro lado, según Rivera, al ser investigadores considerados como seres históricos, debemos tener de antemano nuestros prejuicios durante el acto de investigar pues Gadamer afirma que estos "son el suelo desde el cual comprendemos el mundo y la ciencia no puede ser ciega de sus prejuicios, pues estos son la realidad histórica de nuestro ser". Estos prejuicios son entendidos como metodologías o paradigmas de una época que orientan al investigador y que pueden dirigir el modo de proceder de una investigación, siendo así catalogado el prejuicio como una metodología 22.

Dentro de este pensamiento filosófico de Gadamer también se destacan tres elementos importantes como son la importancia que este le da al lenguaje y a la interpretación para la comprensión de la realidad, la familiarización de estas dos últimas para el desarrollo de 
investigaciones cualitativas, le otorga un carácter epistemológico-filosófico y, finalmente el concepto del círculo hermenéutico, del cual surgen ideas sobre la concepción por parte de la conciencia humana en el cual Gadamer afirma: "no hay que dejarse llevar irreflexivamente por opiniones populares ni por la propia subjetividad".

Partiendo de este último término, Weiss en su análisis habla del círculo hermenéutico como una herramienta útil en la interpretación del fenómeno, puesto que dicho círculo está constituido por varias partes y estas partes constituyen un todo, y en su espiral a medida que va dando vueltas, profundiza y da una interpretación más pertinente de los significados, es así como analizando el fenómeno de estudio desde diferentes partes, se podía llegar a la interpretación de un todo ${ }^{23}$.

En este orden de ideas, para Koskinen en su artículo, refiere que Gadamer define la investigación hermenéutica como un viaje de aprendizaje que no solo está destinado a investigadores clínicos y el objetivo de su aparición es el desarrollo de teorías como de praxis, la cual se enfrenta constantemente a nuevos desafíos ${ }^{24}$.

Un ejemplo de ello puede ser el estudio realizado por Ferro, Rodríguez y Cárdenas en la que a través de un análisis de la historicidad de la formación del estudiante de enfermería y cómo éste construye sus percepciones y significados del cuidado; destaca como el autoconocimiento y reconocimiento del otro en una relación de alteridad y el respeto a la dignidad humana, favorece al desarrollo de la sensibilidad y espiritualidad necesaria dentro del contexto profesional para lograr brindar un cuidado holístico, como ejercicio esencial de la enfermería 25 .

Finalmente, para enfermería, la aplicación de la filosofía Gadameriana contribuye en el sentido de visualizar a los pacientes en un aspecto más amplio en donde se tengan en cuenta su historia, su contexto social, su cultura y sus experiencias, que permitan crear nuevos significados y formar "una fusión de horizontes", que construya nuevos conocimientos. Esto puede implicar nuevos desafíos, ya que, al conocer estas nuevas experiencias, podemos corregir nuestros propios prejuicios o ponerlos en riesgo al realizar dicha fusión al intentar comprender al otro, lo cual es esencial desde el punto de vista fenomenológico ${ }^{26}$.

\section{Conclusiones}

La profesión de enfermería desde sus diferentes ámbitos de desarrollo se ha visto influenciada desde sus inicios por diferentes corrientes filosóficas que han contribuido a su fortalecimiento y crecimiento como disciplina, aportando conocimiento y evidencia científica a la profesión desde diferentes pensadores.

A partir del desarrollo del presente ensayo teórico, permitió discernir la relevancia de la corriente fenomenológica desde Edmund Husserl, Martin Heidegger y Hans-Georg Gadamer los cuales proponen trayectorias y estrategias metodológicas capaces para ser aprovechadas en la investigación cualitativa de enfermería y que a pesar de sus distintas perspectivas tienen el objetivo de interpretar y comprender la esencia del individuo en su propio mundo desde la visión eidética de Husserl, la postura existencialista de Heidegger y la historicidad de Gadamer.

De esta manera, desde el ámbito de la investigación cualitativa en una perspectiva fenomenológica y hermenéutica, se puede llegar a generar una acercamiento efectivo e idóneo a los fenómenos que surgen desde el ejercicio del cuidado de enfermería dentro de una 
realidad social, otorgando las bases y cimientos para el desarrollo de su práctica profesional. Orientado a un cuidado humanizado al visualizar al paciente como un ser humano holístico con sus propias experiencias y vivencias desde una visión intersubjetiva.

Por consiguiente, estas perspectivas contribuyen a que la Enfermería asuma el reto de posicionarse en el campo de la salud como una ciencia, una disciplina y una profesión autónoma y liberal. Dando un cambio fundamental a lo largo de su orientación no solo para tratar de comprender las investigaciones, sino también desarrollar y afianzar la práctica del cuidado más eficaz, es decir, las realidades cuya naturaleza y estructura puedan ser observadas de manera intersubjetiva y con base en las experiencias de la persona que recibe el cuidado.

\section{Referencias}

1. Contreras-de-la Fuente, H.S. y Castillo-Arcos, L.C. 2016. Fenomenología: una visión investigativa para enfermería. Cultura de los Cuidados (Edición digital), 20,44. [Citado 27 May 2021]; Disponible en: http://dx.doi.org/10.14198/cuid.2016.44.02

2. Soto Núñez CA, Vargas Celis IE. La Fenomenología de Husserl y Heidegger. Husserl and Heidegger's phenomenology [Internet]. 2017 [Citado 27 May 2021]; Disponible en: http://rua.ua.es/dspace/handle/10045/69271

3. Ramírez Perdomo Claudia Andrea. Fenomenología hermenéutica y sus implicaciones en enfermería. Index Enferm [Internet]. 2016 Jun [Citado 28 May 2021]; 25(1-2): 82-85. Disponible en: http://scielo.isciii.es/scielo.php?script=sci_arttext\&pid=S1132-12962016000100019\&lng=es.

4. Angulo Marcial, Noel, El ensayo: algunos elementos para la reflexión. Innovación Educativa [Internet]. 2013 [Citado 22 May 2021]; 13(61): 107-121. Disponible en: https://www.redalyc.org/articulo.oa?id=179427877007

5. Guerrero-Castañeda, Raúl Fernando, Ojeda-Vargas, Ma. Guadalupe, LA FENOMENOLOGÍA, Y SU USO EN LA PRODUCCIÓN CIENTÍFICA DE ENFERMERÍA: ESTUDIO BIBLIOMÉTRICO 2010 - 2014. Ra Ximhai [Internet]. 2015 [Citado 21 May 2021]; 11(2): 193-206. Disponible en: https://www.redalyc.org/articulo.oa?id=46143101012

6. Husserl E, Szilasi W. Philosophie als strenge Wissenschaft [Internet]. Libro impreso: Aleman. Frankfurt a.M: Klostermann; 1965. (Quellen der Philosophie: Texte und probleme, 1.). Disponible en: https://www.worldcat.org/title/philosophie-als-strenge-wissenschaft/oclc/21497109

7. Husserl E. La idea de la fenomenología de Edmund Husserl [Internet]. 2016 [Citado 27 May 2021]. Disponible en: https://es.scribd.com/book/316918607/La-idea-de-la-fenomenologia

8. Husserl E. Ideas relativas a una fenomenología pura y una filosofía fenomenológica. J. Gaos, trad. México - Buenos Aires: México: Fondo de Cultura Económica; 1997.

9. Ramírez-Pérez M., Cárdenas-Jiménez M., Rodríguez-Jiménez S. El Dasein de los cuidados desde la fenomenología hermenéutica de Martín Heidegger. Enferm. univ [Internet]. 2015 [Citado 28 May 2021]; 12(3): 144-151. Disponible en: https://doi.org/10.1016/j.reu.2015.07.003

10. Guerrero-Castañeda Raúl Fernando, Prado Marta Lenise Do, Kempfer Silvana Silveira, Ojeda Vargas Ma Guadalupe. Momentos del Proyecto de Investigación Fenomenológica en Enfermería. Index Enferm [Internet]. 2017 Jun [Citado 28 May 2021]; 26(1-2): 67-71. Disponible en: http://scielo.isciii.es/scielo.php?script=sci_arttext\&pid=S1132-12962017000100015\&lng=es.

11. Guillen DEF. Investigación cualitativa: Método fenomenológico hermenéutico. Propósitos Represent. [Internet]. 2019 [Citado 28 May 2021]; 7(1): 201-29. Disponible en: http://www.scielo.org.pe/scielo.php?script=sci_arttext\&pid=S2307-79992019000100010

12. Soto Núñez, C.A., \& Vargas Celis, I.E. La Fenomenología de Husserl y Heidegger. Cultura de los Cuidados [Internet]; 2017 [Citado 28 May 2021]; 21(48). Disponible en: http://dx.doi.org/10.14198/cuid.2017.48.05

13. La Cuesta Benjumea, Carmen de, LA INVESTIGACIÓN CUALITATIVA Y EL DESARROLLO DEL CONOCIMIENTO EN ENFERMERÍA. Texto \& Contexto Enfermagem [Internet]. 2010 [Citado 28 May 2021]; 19(4): 762-766. Disponible en: https://www.redalyc.org/articulo.oa?id=71416100020 
14. Thurnher, R. "iA las cosas mismas!" Acerca de la significación de la máxima fenomenología fundamental en Husserl y Heidegger. Seminarios de filosofía [Internet]. 1996 [Citado 28 May 2021]; 9: 24-44

15. Valderrama O. Aportes de la Fenomenología Heideggeriana al Cuidado Enfermero. Pakamuros [Internet]. 2018 [Citado 28 May 2021]; 6(1): 7. Disponible en: http://revistas.unj.edu.pe/index.php/pakamuros/article/view/57

16. León EA. El giro hermenéutico de la fenomenológica en Martín Heidegger. Polis Rev Latinoam [Internet]. 2009 [Citado 27 May 2021]; (22). Disponible en: http://journals.openedition.org/polis/2690

17. Ramírez-Pérez M., Cárdenas-Jiménez M., Rodríguez-Jiménez S. El Dasein de los cuidados desde la fenomenología hermenéutica de Martín Heidegger. Enferm. univ [Internet]. 2015 [Citado 28 May 2021]; 12(3): 144-151. Disponible en: https://doi.org/10.1016/j.reu.2015.07.003.

18. Herrera $L$, Rivera M. Fundamentos fenomenológicos para un cuidado comprensivo de enfermería. Texto Contexto Enferm. 2006 [Citado 27 May 2021]; $15 . \quad$ Disponible en: https://www.scielo.br/j/tce/a/GqNTJjZyMRPN4BjLmbKJ8Xw/?format=pdf\&lang=es

19. Mendieta-Izquierdo G, Ramírez-Rodríguez JC. La fenomenología desde la perspectiva hermenéutica de Heidegger: una propuesta metodológica para la salud pública. Rev Fac Nac Salud Pública. 2015
[Citado
28 May
2021];
33(3):
435-43.
Disponible
en: http://www.scielo.org.co/pdf/rfnsp/v33n3/v33n3a14.pdf

20. Pérez JJ, Nieto-Bravo JA, Santamaría-Rodríguez JE. La hermenéutica y la fenomenología en la investigación en ciencias humanas y sociales. Civilizar Cienc Soc Humanas [Internet]. 2019 [Citado 27 May 2021]; 19(37): 21-30. Disponible en: https://doi.org/10.22518/usergioa/jour/ccsh/2019.2/a09

21. Schneider FER. Hermenéutica y autoconciencia histórica en el acto de investigar. Andamios Rev Investig Soc [Internet]. 2020 [Citado 27 May 2021]; 17(43): 95-114. Disponible en: https://doi.org/10.29092/uacm.v17i43.766

22. Rodríguez Moya E. La Hermenéutica Gadameriana Como Síntesis Entre El Enfoque Cuantitativo Y Cualitativo En La Investigación Social. GADAMERIAN HERMENEUTICS Synth Quant Qual APPROACH Soc Res. [Internet]. 2019 [Citado 27 May 2021]; (14): 1-9. Disponible en: http://dx.doi.org/10.4067/s0718-50652019000100204

23. Weiss E. Hermenéutica Y Descripción Densa Versus Teoría Fundamentada. Rev Mex Investig Educ. abril de 2017;22(73):637-54.

24. Koskinen C, Nyström L. Hermeneutic application research - finding a common understanding and consensus on care and caring. Scand J Caring Sci. [Internet]. 2017 [Citado 27 May 2021]; 31(1): 175-82. Disponible en: https://pubmed.ncbi.nlm.nih.gov/26712586/

25. Ferro-Rivera, N., Rodríguez-Jiménez, S., Cárdenas-Jiménez, M., Significados sobre el arte del cuidado desde la perspectiva de los estudiantes de Licenciatura en Enfermería. Enfermería Universitaria [Internet]. 2018 [Citado 25 May 2021]; 15(4): 402-415. Disponible en: https://www.redalyc.org/articulo.oa?id=358758714009

26. Midtbust, M.H., Alnes, R.E., Gjengedal, E. et al. A painful experience of limited understanding: healthcare professionals' experiences with palliative care of people with severe dementia in Norwegian nursing homes. BMC Palliat Care [Internet]. 2018 [Citado 28 May 2021]; 17(25). Disponible en: https://www.ncbi.nlm.nih.gov/pmc/articles/PMC5809908/

\section{Editor}

Escuela de Enfermería de la Universidad Católica Santo Toribio de Mogrovejo, Chiclayo, Perú

\section{Cómo citar este trabajo}

Acevedo-Figueroa L, Agüero-Grande J, Oyola-Lamprea A, Trujillo-León Y. Contribuciones significativas de la fenomenología de Edmund Husserl, Martin Heidegger y Hans-Georg Gadamer a la investigación en enfermería. Acc Cietna: para el cuidado de la salud [Internet]. 2021; 8(2): 85 - 94. Disponible en: https://doi.org/10.35383/cietna.v8i2.686

\section{Financiación}

El presente artículo no cuenta con financiación específica de agencias de financiamiento en los sectores público o privado para su desarrollo y/o publicación. 


\section{Conflicto de interés}

Los autores del artículo declaran no tener ningún conflicto de intereses en su realización.

(c) Los autores. Este artículo es publicado por la Revista Acc Cietna: para el cuidado de la salud de la Escuela de Enfermería, Universidad Católica Santo Toribio de Mogrovejo.

Este es un artículo de acceso abierto, distribuido bajo los términos de la Licencia Creative Commons Atribución-NoComercial-CompartirIgual 4.0 Internacional (CC BY-NC-SA 4.0), que permite el uso no comercial, distribución y reproducción en cualquier medio, siempre que la obra original sea debidamente citada. 